\title{
Microcomputer-Based Quantification of Maize Streak Virus Symptoms in Zea mays
}

\author{
Darrin P. Martin and Edward P. Rybicki
}

Microbiology Department, University of Cape Town, Private Bag, Rondebosch, Western Cape, South Africa 7701. Accepted for publication 20 January 1998.

\begin{abstract}
Martin, D. P., and Rybicki, E. P. 1998. Microcomputer-based quantification of maize streak virus symptoms in Zea mays. Phytopathology 88: 422-427.

We investigated the use of computer-assisted image analysis techniques for the objective quantification of maize streak virus (MSV) symptoms in Zea mays. We compared independent duplicate evaluations of chlorotic lesion areas occurring on MSV-infected leaves using visual assessment, a commercial image analysis system, and a custom image analysis system employing software developed in our laboratory. Rela-

estimations. The commercial image analysis system afforded no significant improvement in precision or accuracy over the custom system. An important advantage of examining images using the custom-written software was that the software permitted a high degree of analysis automation. Digitized images of maize leaves could be automatically analyzed by the custom software five times faster than, and with the same precision and accuracy as, when the same images were analyzed with the commercial software. Because of the flexibility of the image analysis techniques described, they should be applicable to the measurement of symptom severity in other plant host-pathogen combinations.
\end{abstract} tive to visual assessments of disease severity, computer-assisted image analysis employing both the commercial and custom systems provided significant enhancements in the accuracy and precision of chlorotic area
Additional keywords: disease assessment reliability, leaf tracing, pixel thresholding, source code.
Quantification of disease severity is central to the evaluation of gross host-pathogen interactions in plant pathology and breeding. Methods used in the quantification of disease severity should ideally be simple, cheap, fast, flexible, precise, and accurate. Currently employed methods of symptom assessment for many plant diseases are either qualitative or only semiquantitative and often rely heavily on visual assessment by a single scorer. While many of these techniques have been successfully employed in the assessment of disease control practices and the identification of disease-resistant plant varieties, various studies have demonstrated the unreliability of the human eye for the objective determination of symptoms $(5,8,11,16)$. These studies have found that data based on visual assessment of disease symptoms are inaccurate when percent leaf injury is close to $50 \%$ (5) and contain biases correlated with the numbers $(16)$, areas $(11,16)$, and shapes of disease lesions $(8,17)$.

Almost since their inception, personal computers have been employed in the analysis of plant disease severity $(1,8)$. The response of a video camera to incident light is unaffected by the amounts of disease injury, and objective computational quantification of diseased areas is not prone to biases related to lesion number and shape (8). Many commercial image analysis and processing systems, often backed by powerful software, are currently available and are being extensively used in plant disease assessment $(7,9,12,15)$. An important factor in the use of these dedicated systems is, however, their high cost. Depending on the complexity of the disease assessment task at hand, cheap alternative protocols may be devised using the personal computers and digital image capture equipment commonly found in many laboratories and homes $(6,8,14)$. Useful in this regard is the availability, via the Internet, of a wealth of rel-

Corresponding author: E. P. Rybicki; E-mail address: ed@molbiol.uct.ac.za

Publication no. P-1998-0316-01R

(C) 1998 The American Phytopathological Society atively sophisticated image processing and analysis software that is, in most cases, inexpensive and often free.

Maize streak virus (MSV) is the causal agent of maize streak disease, which is responsible for devastating maize losses throughout Africa (2). The virus produces well-defined chlorotic lesions on the leaves of afflicted host plants that also include barley, wheat, and a number of grasses (2). For breeding purposes, the severity of maize streak disease in individual plants is currently assessed using semiquantitative five- or ten-point systems $(2,3,13)$. However, MSV pathology studies in our laboratory require more precise differentiation among symptoms in differentially resistant maize cultivars infected with differentially virulent MSV strains, which has necessitated the development of an accurate quantitative technique for MSV symptom determination.

In the current study, we investigated the suitability of computerbased image analysis techniques for the objective quantification of maize streak symptoms in Zea mays. Approximations of percent chlorotic areas occurring on MSV-infected maize leaves were made both visually and with image analysis techniques. The latter techniques involved the use of both a commercial image analysis system and a custom-developed system using software written in our laboratory. The symptom assessment techniques investigated were compared for accuracy, precision, and requisite analysis time.

\section{MATERIALS AND METHODS}

Virus strains. Agroinfectious constructs of differentially virulent MSV strains were either produced in this laboratory (MSVMat, MSV-Kom, MSV-Set, MSV-Tas, MSV-VM, and MSV-VW; D. P. Martin, W. Schnippenkoetter, J. A. Willment, and E. P. Rybicki, unpublished data) according to Grimsley et al. (4) or obtained from M. Boulton (MSV-N; John Innes Centre, Norwich, United Kingdom).

Specimen preparation. Inoculation of 3-day-old seedlings of the MSV-sensitive Zea mays cultivar Jubilee was carried out by agroinfection (4). Fifteen days after inoculation, blades of the third 
leaves of 6 uninfected control plants and 110 plants displaying symptoms ranging from almost complete chlorosis to small isolated chlorotic leaf spots were cut transversely into four segments of equal length. Color photographs were taken of the second segment from the base of each leaf blade. Once photographed, leaf segments were wrapped in damp paper towels and stored on ice until use.

Visual analysis of symptoms. Visual assessment of symptoms was carried out on all the leaf segments involved in the study. Percent chlorotic area estimates were made from projections of the color slide photographs taken during preparation of the leaf segments. Four scorers, three of whom had extensive experience in visually assessing maize streak symptoms, independently estimated percent chlorotic areas of the leaf segments under identical conditions. Each scorer repeated their visual assessment of chlorotic areas twice on consecutive weeks.

Image capture systems. Image analysis in this study utilized both a Joyce Loebl IV120 image analysis system (JL Automation Ltd., Sunderland, United Kingdom) and GDS 5000 image capture equipment (Ultra-Violet Products, Upland, CA). The commercial Joyce Loebl system comprised a Hitachi KP-140 monochrome video camera (Hitachi Denshi Ltd., Tokyo) connected to a Joyce Loebl control processor. Near-uniform top lighting was supplied by two fluorescent lamps. The GDS 5000 image capture equipment used in the custom image analysis system comprised an UltraViolet Products monochrome video camera connected to an Image Store 5000 processor box (Ultra-Violet Products). Two opaque 100 -W light bulbs arranged to provide near-uniform top lighting were used for specimen illumination.

Image analysis protocols. Between 15 and 18 leaf segments, including the six symptomless control leaves, were grouped in the same orientation without touching one another on black felt within a $15 \times 25-\mathrm{cm}$ area. Images of leaf segments containing 256 gray shades with dimensions of $512 \times 512$ pixels and $756 \times 512$ pixels were captured with the Joyce Loebl and GDS 5000 systems, respectively. Also included in every image was an internal color control comprising a contact print of a photographic graded gray scale or "step-wedge" (Fig. 1) (Stouffer Graphic Arts, Inc., South Bend, IN). Optimum video camera aperture settings permitting the widest possible spread of pixel colors in captured images were established based on the examination of pixel color frequency histograms from images containing a series of step-wedges and a test group of leaf segments with MSV symptoms ranging from mild to severe. These images were captured using a variety of aperture settings with constant top lighting. Establishment of an optimum lighting intensity standard exploited the phenomenon of image flooding (which results in uniform whitening of bright areas of an image that are excessively illuminated) of a selected step-wedge that was then employed as a standard lighting intensity indicator in all future analyses with both of the image analysis systems used.

A pixel thresholding strategy, based on that described by Lindow and Webb (8), was used for analysis of images captured with both the Joyce Loebl and GDS 5000 systems. Images obtained with the Joyce Loebl system were analyzed using JLGenias (version 3.01; JL Automation Ltd., Sunderland, United Kingdom), a commercial software package designed specifically for use with this image analysis system. Analysis was partially automated with the use of a custom "macro"-based analysis sequence. With the custom system, captured images were analyzed using Image09, an image analysis program written specifically to set pixel threshold values automatically based on the shade of designated step-wedge segments (available online from the University of Cape Town). Image capture and analysis of leaf segments using the commercial system was carried out by a single scorer with extensive experience in the use and development of image analysis techniques. Image capture and analysis of leaf segments using the custom system was carried out independently by the same four scorers that visually assessed chlorotic areas. Prior to image capture and analysis, scorers received approximately 5 min of instruction on setting up the image capture equipment and using the Image09 software. For both the custom and commercial systems, scorers repeated the entire image capture and analysis sequence twice.

The degree of analysis automation achievable with Image09 was investigated. For images examined using the custom image analysis system, the pixel color values corresponding to both the background/healthy leaf area and the healthy/chlorotic leaf area threshold cut-off values determined automatically by Image09 were compared with corresponding values manually selected by the four scorers. To assess the feasibility of using Image09 to determine thresholding cut-off values without any human input, the software was used to analyze all of the images captured with the Joyce Loebl system without any scorer input other than image selection. Automatically determined threshold cut-off values, percent chlorotic leaf areas, and analysis times were recorded.

The mean chlorotic area values of the asymptomatic control leaf segments included in each analyzed image were subtracted from the chlorotic area estimate for each symptomatic leaf segment analyzed in that image. This yielded chlorotic area estimates adjusted to account for pixels incorrectly grouped in the chlorotic leaf area category.

Analysis of symptoms by leaf tracing. A hybrid of analogue and digital techniques based on the acetate image analysis procedure described by Nutter et al. (10) was used for quantification of the actual chlorotic areas of a random subset of the leaf segments involved in this study. For each selected leaf segment, 5- to 10-fold-
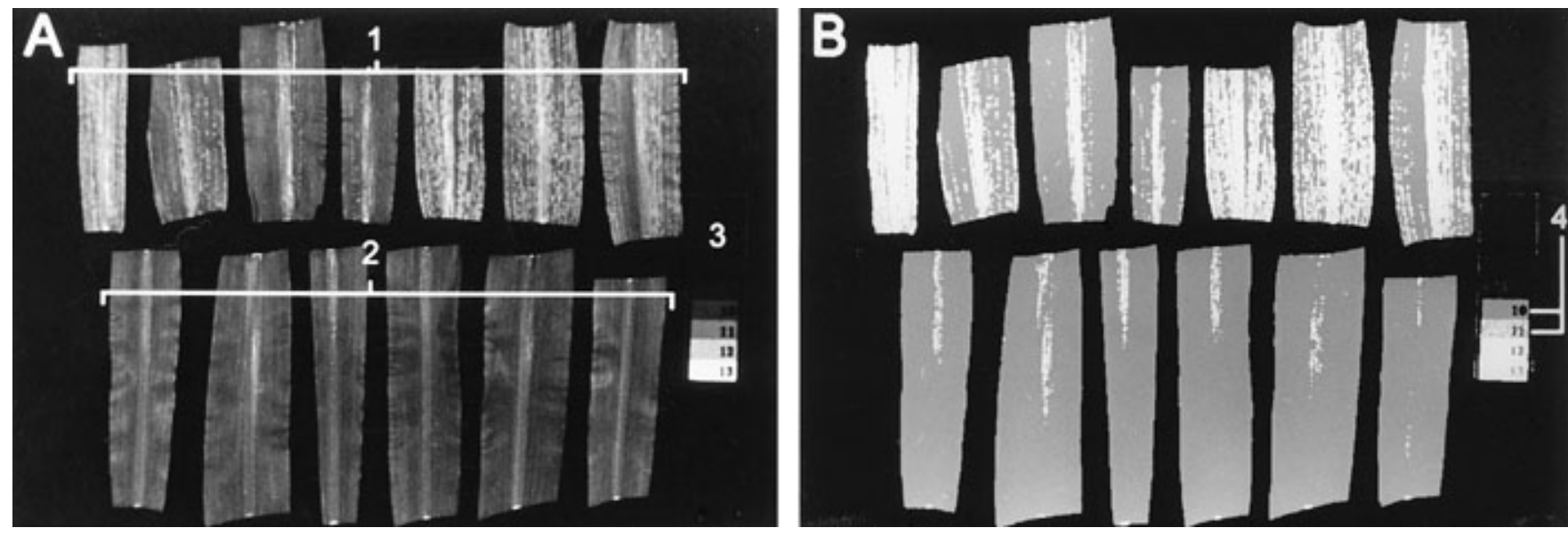

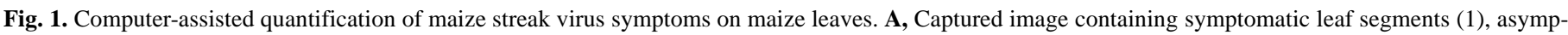

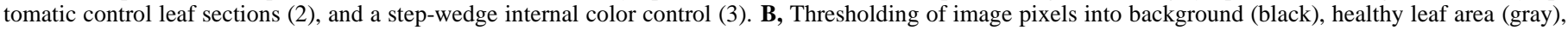
and chlorotic leaf area (white) categories based on the gray shades of specific step-wedge segments (4). 
magnified tracings from projections of color slides were made. Analysis of a single leaf segment involved making tracings of both total leaf area and total area of achlorotic leaf tissue. A digital image containing these was captured using the GDS 5000 system. Each image was processed to obtain a two-color (black and white) image using Paint Shop Pro version 4.12 (JASC Inc., Eden Prairie, $\mathrm{MN})$. Calculation of percent chlorotic leaf area from a processed image was carried out using IMPROCES version 4.2 (John Wagner, San Diego, CA) to separately count the number of black pixels comprising the total and achlorotic leaf area tracings. For each selected leaf segment, tracings of total and achlorotic areas and assessment of percent chlorotic areas were repeated twice on consecutive weeks.

Assessment of symptom quantification techniques. Linear regression analysis as described by Nutter et al. (10) was used to estimate the relative precision of all the analysis techniques described. Reproducibility of chlorotic area estimations using visual symptom assessment and the image analysis techniques was determined for each scorer by regressing their first and second chlorotic area ratings of leaf segments against one another. To determine interscorer precision of symptom assessments made visually and with the custom image analysis system, means of each scorer's duplicated chlorotic area estimates were linearly regressed against those of all the other scorers and analyzed accordingly. The accuracy of individual scorers employing the image analysis and visual assessment techniques was determined by linear regression analysis of the means of duplicated chlorotic area estimates against the means of duplicated percent chlorotic area measurements made using the leaf tracing technique. To determine the practical implications of employing the different symptom quantification approaches, we analyzed visual and image analysis-derived chlorotic area estimates in relation to the seven MSV strains used. The mean percent chlorotic areas of leaves infected with the different MSV strains as determined by the four scorers visually and with the custom image

TABLE 1. Determination of intrascorer precision by linear regression analysis of repeated chlorotic area estimates obtained using the various symptom assessment techniques

\begin{tabular}{llccc}
\hline & & \multicolumn{3}{c}{ Regression parameters } \\
\cline { 4 - 5 } Analysis technique & Scorer & $R^{2}$ & Slope & y-Intercept \\
\hline Leaf tracing & Manual & 0.990 & 0.976 & 1.140 \\
Commercial image analysis & Manual & 0.967 & 1.000 & 1.376 \\
& Automatic & 0.972 & 0.990 & 1.360 \\
Custom image analysis & Scorer 1 & 0.966 & 1.019 & -1.270 \\
& Scorer 2 & 0.962 & 0.965 & 1.031 \\
& Scorer 3 & 0.972 & 0.999 & 0.033 \\
Visual assessment & Scorer 4 & 0.969 & 0.979 & 0.539 \\
& Scorer 1 & 0.946 & 0.955 & $5.705^{* a}$ \\
& Scorer 2 & 0.965 & 0.995 & $-3.023^{*}$ \\
& Scorer 3 & 0.945 & 0.983 & $2.819^{*}$ \\
& Scorer 4 & 0.896 & $1.087 * \mathrm{~b}$ & 0.999 \\
\hline
\end{tabular}

a $y$-Intercept values that are significantly different from $0(P \leq 0.01)$ are followed by *.

b Slope values that are significantly different from $1(P \leq 0.01)$ are followed by *. analysis system were compared. For all the analysis techniques, total times taken to obtain chlorotic area estimates were recorded and averaged to obtain mean analysis times per leaf segment.

\section{RESULTS}

Assessment of precision. In repeated chlorotic area assessments by single scorers (Table 1), leaf tracing had the highest reproducibility of the techniques evaluated. While repeated visual assessment of chlorotic areas by individual scorers yielded the least reproducible results, the repeatability of a single scorer's chlorotic area estimates using either the commercial or custom image analysis system were similar. Linear regression analysis of repeated symptom assessments by all scorers using either image analysis system yielded regression lines with y-intercepts and slopes that did not differ significantly from 0 and 1, respectively. Although linear regressions of repeated visual symptom estimations yielded regression lines with slopes that did not differ significantly from 1 , for all scorers except scorer 4 , y-intercepts of the regression lines differed significantly from 0 .

The interscorer precisions of chlorotic area estimates were generally higher using the custom image analysis system than when estimates were made visually (Table 2). In all cases, when estimates were made using the image analysis technique, y-intercepts and slopes of regression did not differ significantly from 0 and 1, respectively. Despite generally high coefficients of determination when regressing visually determined estimates of different scorers against one another, all of the y-intercepts and four of the six slopes derived from these regressions were significantly different from 0 and 1 , respectively.

Assessment of accuracy. Regression analysis of estimated against actual chlorotic areas indicated that the commercial and custom image analysis systems were comparable in accuracy (Table 3 ).

TABLE 3. Determination of disease assessment technique accuracy based on linear regression analysis of chlorotic area estimates obtained using the various analysis techniques plotted against actual chlorotic areas

\begin{tabular}{llccc}
\hline \multirow{2}{*}{ Analysis technique } & & \multicolumn{3}{c}{ Regression parameters } \\
\cline { 3 - 5 } Commercial image analysis & Scorer & $R^{2}$ & Slope & y-Intercept \\
\hline \multirow{2}{*}{ Custom image analysis } & Automatic & 0.976 & 1.040 & -0.877 \\
& Scorer 1 & 0.985 & 1.045 & 0.346 \\
& Scorer 2 & 0.971 & 1.032 & -0.782 \\
& Scorer 3 & 0.980 & 1.020 & -0.190 \\
Visual assessment & Scorer 4 & 0.979 & 0.999 & 0.610 \\
& & & & \\
& Scorer 1 & 0.913 & $1.066^{* a}$ & $12.958^{* b}$ \\
& Scorer 2 & 0.960 & $1.245^{*}$ & 1.064 \\
& Scorer 3 & 0.957 & $1.135^{*}$ & $5.201^{*}$ \\
& Scorer 4 & 0.927 & 1.029 & $8.385^{*}$ \\
\hline
\end{tabular}

${ }^{a}$ Slope values that are significantly different from $1(P \leq 0.01)$ are followed by *.

${ }^{\mathrm{b}} \mathrm{y}$-Intercept values that are significantly different from $0(P \leq 0.01)$ are followed by *.

TABLE 2. Determination of interscorer precision by linear regression analysis of chlorotic area estimates made by different scorers both visually and using the improvised image analysis system

\begin{tabular}{|c|c|c|c|c|c|c|}
\hline \multirow[b]{2}{*}{ Regression comparison } & \multicolumn{3}{|c|}{ Visual assessment } & \multicolumn{3}{|c|}{ Custom image analysis system } \\
\hline & $R^{2}$ & Slope & $\mathrm{y}$-Intercept & $R^{2}$ & Slope & $\mathrm{y}$-Intercept \\
\hline Scorer 1 vs. Scorer 2 & 0.932 & $1.098^{* \mathrm{a}}$ & $-9.237 * b$ & 0.948 & 0.983 & 0.948 \\
\hline Scorer 1 vs. Scorer 3 & 0.929 & 0.994 & $-3.028 *$ & 0.960 & 1.030 & -0.841 \\
\hline Scorer 2 vs. Scorer 3 & 0.983 & $0.900 *$ & $5.621^{*}$ & 0.953 & 0.953 & 0.034 \\
\hline Scorer 2 vs. Scorer 4 & 0.937 & $0.780 *$ & $11.656 *$ & 0.952 & 0.996 & 0.727 \\
\hline Scorer 3 vs. Scorer 4 & 0.972 & $0.876^{*}$ & $6.371 *$ & 0.962 & 0.962 & 1.319 \\
\hline
\end{tabular}

a Slope values that are significantly different from $1(P \leq 0.01)$ are followed by $*$.

${ }^{\mathrm{b}} \mathrm{y}$-Intercept values that are significantly different from $0(P \leq 0.01)$ are followed by $*$. 
In all cases, the slopes and y-intercepts from these regressions did not differ significantly from 1 and 0 , respectively (Fig. 2). Conversely, regressions of visual estimates against actual chlorotic areas yielded both y-intercepts that were significantly greater than 0 for scorers 1,3 , and 4 and slopes that were significantly greater than 1 for scorers 1,2 , and 3 (Table 3 ).

When comparing the mean percent chlorotic areas of leaves infected with the different MSV strains as determined by the four scorers visually and with the custom image analysis system, it was noted that for all of the MSV strains, except MSV-VM, visually derived estimates were higher than estimates obtained by image analysis (Fig. 3). In all cases, except for MSV-VW, there was less variation between the mean chlorotic area estimates of the scorers when image analysis was used. Results obtained by image analysis indicated that, in terms of average chlorotic areas resulting from infection, MSV-Kom and MSV-VW did not differ significantly from one another in severity, but both were significantly less severe than MSV-N. Alternatively, results obtained by visual assessment indicated that the severities of MSV-N and MSV-Kom were not significantly different from one another, but that both were significantly more severe than MSV-VW.

Assessment of automated symptom quantification. Of the 112 images captured using the custom image analysis system and analyzed using Image09, the step-wedge was correctly identified in $111(99.1 \%)$, the segment corresponding to healthy leaf area was correctly identified in $110(98.2 \%)$, and the segment corresponding to chlorotic leaf area was correctly identified in 108 $(96.4 \%)$. For the background/healthy and healthy/chlorotic leaf area pixel thresholding cut-off values, the mean absolute difference between the manually and automatically determined values for all scorers were $2.3( \pm 2.3$ standard deviation [SD]) and 4.4 ( $\pm 4.0 \mathrm{SD})$, respectively. Of the 28 images captured using the commercial system and examined with Image09, the step-wedge was successfully detected and used to set thresholding cut-off values in

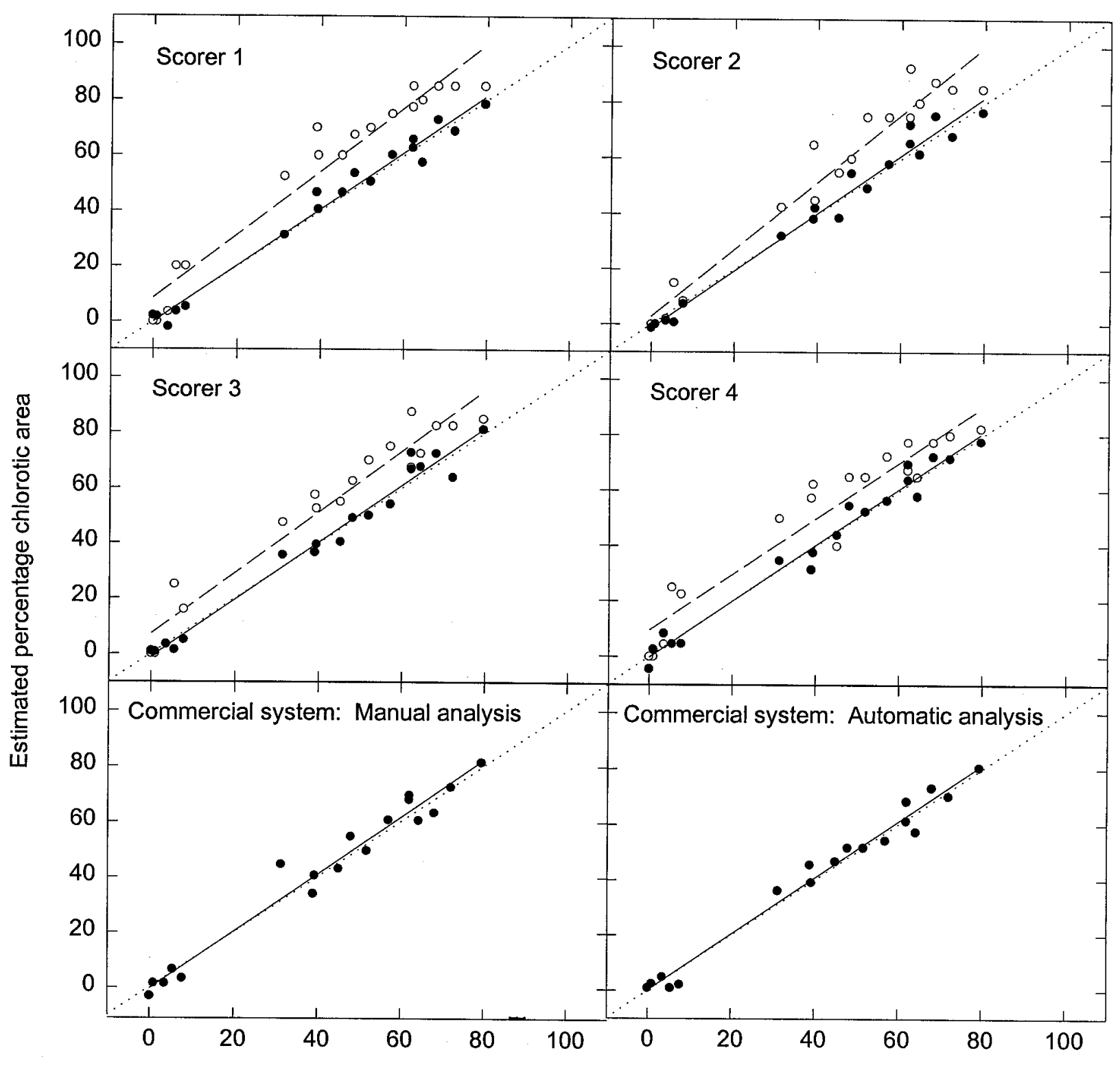

Actual percentage chlorotic area

Fig. 2. Regression of image analysis ( $\bullet$ and solid line) and visually derived ( $O$ and dashed line) percent chlorotic area estimates against actual percent chlorotic areas of 20 randomly selected leaf sections. Image analysis carried out by scorers 1, 2, 3, and 4 employed a custom system. Images captured using a commercial image analysis system were analyzed both manually using the commercial software (JLGenias, version 3.01) and automatically using custom-written software (Image09). The dotted lines have slopes $=1$ and $y$-intercepts $=0$. The coefficients of determination, y-intercepts, and slopes of the regressions presented here are given in Table 3. 
$27(96.4 \%)$. Linear regression analysis of chlorotic area estimates from the first and second groups of images captured with this system indicated that automatic thresholding and symptom calculation was at least as precise as that carried out manually on the same images using JLGenias, the commercial image analysis software (Table 1). The means of duplicated chlorotic area estimates obtained manually using the commercial software and automatically using Image09 were highly correlated and, when regressed against one another, yielded a regression line with a y-intercept of $-1.453(P>0.99$ that this value is not significantly different from $0)$, a slope of $1.025(P>0.99$ that this value is not significantly different from 1), and a coefficient of determination of 0.990. The accuracy of chlorotic area estimates determined automatically with Image09 compared very favorably with those determined manually using the commercial software (Fig. 2).

Duration of analyses. Of the techniques assessed, visual quantification of symptoms was fastest, requiring, on average, 7.6 $( \pm 2.6 \mathrm{SD}) \mathrm{s}$ per leaf segment. The leaf tracing technique, which required $35.7( \pm 15.4 \mathrm{SD})$ min per leaf segment, was the most timeconsuming. Mean analysis times required to manually determine the percent chlorotic areas on individual leaf segments using the custom and commercial image analysis systems were $36.2( \pm 2.6 \mathrm{SD})$ and $52.9( \pm 6.1 \mathrm{SD}) \mathrm{s}$, respectively. Automated analysis of images captured with the commercial system using Image09 required only $10.1( \pm 0.3 \mathrm{SD}) \mathrm{s}$ per leaf.

\section{DISCUSSION}

We demonstrated the potential use of commonly available resources for the development of a fast, precise, accurate, and objective symptom quantification technique. The pixel thresholding approach we used for the analysis and quantification of percent chlorotic leaf areas has been the basis of most published protocols for symptom quantification by image analysis $(1,6,8,9,12)$. Because of the simplicity of pixel thresholding, it was possible to devise a variety of symptom quantification techniques utilizing software either obtained at trivial cost via the Internet or written specifically to suit our purposes. Here, we present an example of one of these techniques together with an analysis of its precision and accuracy relative to both commercial image analysis and visual symptom quantification techniques.

Two components of precision have been identified as significant when attempting to establish the overall precision of disease symptom quantification techniques. These are (i) intrascorer precision, which refers to the reproducibility of disease severity estimates when made by a single scorer on different occasions, and (ii) interscorer precision, which refers to the reproducibility of symptom severity estimates when made independently by two different scorers (10).

Using linear regression analysis, we demonstrated that our custom image analysis technique yielded percent chlorotic area estimates that were both comparable for intrascorer precision with estimates obtained using a commercial image analysis system, and significantly better for intra- and interscorer precision than visual estimates. Regression analysis indicated that, while relative differences between the estimated chlorotic leaf areas remained consistent within the two groups of visual chlorotic area estimates made by a single scorer, there were generally systematic differences between the estimates made by three of the four scorers.

The accuracy of our custom image analysis system was similar to that of the commercial system. Regression analysis revealed that there were neither constant nor systematic errors in estimation related to actual chlorotic areas. All scorers estimated chlorotic areas significantly more accurately with the custom system than when estimating chlorotic areas visually. While the overall accuracy of the visual estimates was high relative to previous reports for other diseases $(10,11,12,16)$, regression analysis showed that visual estimations of chlorosis significantly exceeded actual areas across the entire range of symptoms for three out of four scorers, a situation commonly reported $(11,12,16)$. In addition, and in accordance with previous reports, the degree of overestimation by three out of four scorers increased significantly as actual chlorotic areas increased $(12,16)$. Despite these relatively consistent errors, however, examination of residuals (differences between estimated and actual chlorotic areas) indicated that no mathematical transformation of the visual estimates to achieve greater linearity in the regressions was warranted. Also, the slopes of the regression lines did not differ from 1 in a consistent manner, indicating that no single mathematical transformation to linearity could be uniformly applied to the chlorotic area estimates made by all scorers.

It was found that differentiating between strains based on of the degree of chlorosis they induced in infected plants was influenced by whether the symptoms had been assessed visually or with image analysis. This indicates the need for accurate symptom quantification in studies dealing with the differentiation among MSV strains that have subtly different pathologies.

An important feature of the image analysis protocols employed in this study was the inclusion of healthy leaf segments and a graded

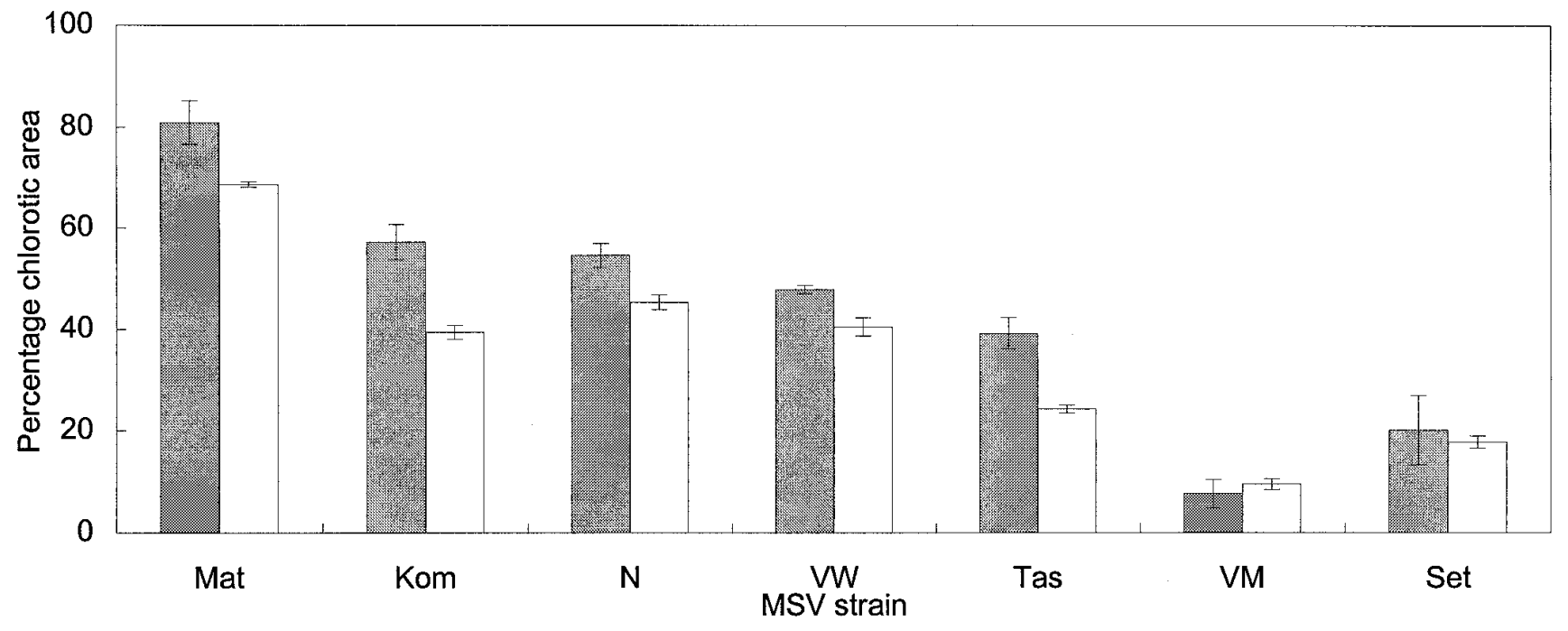

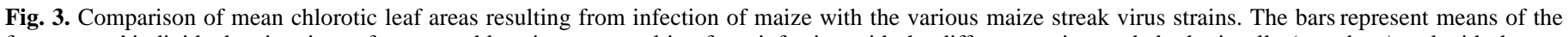

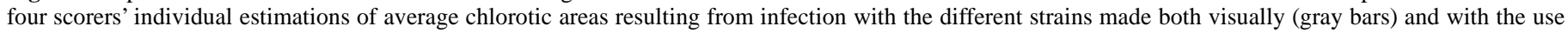
of our custom image analysis system (white bars). The error bars represent standard deviations of the average chlorotic area estimates made by the scorers. 
gray-scale step-wedge as internal controls in every digital image examined. The inclusion of at least six asymptomatic control leaves in all the captured images permitted estimation of the number of pixels grouped in the chlorotic category that should have been grouped in the healthy category; these pixels generally represented the lightly colored leaf midrib. The background level of incorrectly identified chlorosis on healthy leaves was used to correct chlorotic area estimates of symptomatic leaves to discount leaf midribs included in the diseased pixel group.

The step-wedge played a dual role in the image analysis procedures, functioning both as a lighting standard and as an internal color control. Its use obviated the need to specify camera aperture settings and location of lighting source(s) relative to specimens, since these would not be relevant to any system other than our own. With both the custom and commercial systems, we demonstrated the potential usefulness of an incident lighting intensity standard for recreating consistent lighting under different conditions. This represents a viable solution to the problem of standardizing image analysis protocols in different locations using varied equipment and software.

Inclusion of the step-wedge as an internal color control in images also provided a disease symptom independent guide for both the manual and automatic setting of pixel threshold cut-off values. With the use of a step-wedge as an internal color control, coupled with the inclusion of step-wedge identification and examination algorithms in the Image09 software, we demonstrated the potential for negating the input of a human scorer at the analysis stage of symptom assessment. Pixel threshold cut-off values automatically determined by the custom-written software compared well with those specified manually by scorers. Similarly, images captured with the commercial system and analyzed with Image09 without any scorer input other than image selection yielded chlorotic area estimates that were as precise and accurate as chlorotic area estimates obtained manually using JLGenias, the analysis software developed specifically for the commercial system. This result is significant in that it demonstrates that human judgment, which is an important subjective element in any analysis, need not be relied upon to determine which pixels should be grouped in the background, healthy, and diseased categories.

Image 09 has been developed with a built-in calibration capacity enabling the software to automatically determine pixel cut-off values from the graded gray-scale segments of any step-wedge. Therefore, use of this software in conjunction with an appropriate step-wedge will enable increased standardization and automation of analysis in image analysis protocols for quantifying the symptoms of other plant pathogens. We also facilitated direct customization of the software by making the source code, written in Visual Basic and Visual C++ (Microsoft Corporation, Redmond, WA), freely available (available online from the University of Cape Town). It should be possible for anybody reasonably competent at programming in BASIC or C to customize the program to accommodate any foliar disease symptom assessment task. These customizations could include (i) adaptation of the software to analyze symptoms in direct conjunction with image capture equipment; (ii) adding symptom identification algorithms that are more complex than the pixel thresholding ones that are currently in wide use; and (iii) modification of the pixel examination and manipulation algorithms to permit the analysis of color images.

Having ascertained that the image analysis protocols that we developed are suitable for the accurate and precise estimation of chlorotic areas in MSV-infected maize leaves, we are currently employing these protocols to both analyze the virulence of MSV isolates and rapidly screen maize cultivars for MSV resistance. With regard to the analysis of MSV symptoms in pathology studies, the image analysis techniques described here could provide either a viable alternative to, or a valuable refinement of, the semiquantitative MSV symptom rating scales currently employed for breeding purposes $(2,3,13)$.

\section{ACKNOWLEDGMENTS}

We thank J. A. Willment, D. Cox, and J. S. Passmore for being our scorers; T. Sewell for help with the commercial image analysis system; J. S. Passmore and E. van der Walt for proofreading; and the Foundation for Research Development for funding.

\section{LITERATURE CITED}

1. Blanchette, R. A. 1982. New technique to measure tree defect using an image analyzer. Plant Dis. 66:394-397.

2. Efron, Y., Kim, S. K., Fajemisin, J. M., Mareck, J. H., Tang, C. Y., Dabrowski, Z. T., Rossel, H. W., Thottappilly, G., and Buddenhagen, I. W. 1989. Breeding for resistance to maize streak virus: A multidisciplinary team approach. Plant Breed. 103:1-36.

3. Govinden, N., and Rummun, K. 1996. Registration of MSIRI 3B streakresistant maize germplasm. Crop Sci. 36:824

4. Grimsley, N., Hohn, T., Davies, J. W., and Hohn, B. 1987. Agrobacterium-mediated delivery of infectious maize streak virus in maize plants. Nature 325:177-179.

5. Horsfall, J. G., and Barratt, R. W. 1945. An improved grading system for measuring plant diseases. (Abstr.) Phytopathology 35:655.

6. Kampmann, H. H., and Hansen, O. B. 1993. Using colour image analysis for quantitative assessment of powdery mildew on cucumber. Euphytica 79:19-27.

7. Kokko, E. G., Conner, R. L., Kozub, G. C., and Lee, B. 1993. Quantification by image analysis of subcrown internode discoloration in wheat caused by common root rot. Phytopathology 83:976-981.

8. Lindow, S. E., and Webb, R. R. 1983. Quantification of foliar plant disease symptoms using microcomputer-digitized video image analysis. Phytopathology 73:520-524.

9. Newton, A. C. 1989. Measuring the sterol content of barley leaves infected with powdery mildew as a means of assessing partial resistance to Erysiphe graminis f.sp. hordei. Plant Pathol. 38:534-540.

10. Nutter, F. W., Jr., Gleason, M. L., Jenco, J. H., and Christians, N. C. 1993. Assessing the accuracy, intra-rater repeatability, and inter-rater reliability of disease assessment systems. Phytopathology 83:806812 .

11. Parker, S. R., Shaw, M. W., and Royle, D. J. 1995. The reliability of visual estimates of disease severity on cereal leaves. Plant Pathol. 44:856-864.

12. Price, T. V., Gross, R., Ho Wey, J., and Osborne, C. F. 1993. A comparison of visual and digital image-processing methods in quantifying the severity of coffee leaf rust (Hemileia vastatrix). Aust. J. Exp. Agric. 33:97-101.

13. Rodier, A., Assiv, J., Marchand, J.-L., and Hervv, Y. 1995. Breeding maize lines for complete and partial resistance to maize streak virus (MSV). Euphytica 81:57-70.

14. Sah, D. N., and Fehrmann, H. 1992. Virulence patterns of geographically differing isolates of Pyrenophora tritici-repentis and sources of resistance in wheat. Plant Dis. 76:712-716.

15. Shaw, M. W., and Royle, D. J. 1989. Estimation and validation of a function describing the rate at which Mycosphaerella graminicola causes yield loss in winter wheat. Ann. Appl. Biol. 115:425-442.

16. Sherwood, R. T., Berg, C. C., Hoover, M. R., and Zeiders, K. E. 1983. Illusions in visual assessment of Stagonospora leaf spot of orchardgrass. Phytopathology 73:173-177.

17. Webb, R., and Lindow, S. E. 1981. Evaluation of Ascochyta pteridium as a potential biological control agent of bracken fern. (Abstr.) Phytopathology 71:911. 